\title{
Ensemble Trajectory Simulation of Large Jellyfish in the Yellow and Bohai Sea
}

Lingjuan Wu , Jiangling Xu

North China Sea Marine Forecast Center of State Oceanic Administration,266061, Qingdao, China,

\begin{abstract}
Combining Lagrange particle tracking method with the ensemble forecasting concept, one ensemble trajectory model is developed for large jellyfish with vertical movement of jellyfish taken into account in the Yellow and Bohai Sea. Besides, this ensemble model can give quick simulation of drifting trajectories and potential affected range. The comparison between observation and simulation of jellyfish trajectories in the coastal waters of Qingdao and Qinhuangdao as the typical place of the Yellow Sea and Bohai Sea has been made. And the results indicate that the ensemble trajectory model is more reliable than single one, and could provide more useful information for emergency response of jellyfish bloom, especially under the circumstance that autonomic (vertical) movement of of jellyfish and its mechanism is not clear.
\end{abstract}

\section{Introduction}

Due to the global change and human activities, the occurrence frequency and disaster category of large jellyfish increase continuously [1-3]. In recent years, jellyfish has been blooming in the coastal waters of Qingdao and Qinhuangdao including dominant species such as Nemopilema nomurai, Aurelia aurita and so on. According to their occurrence mechanism, these jellyfish could be divided into two types: one is foreign type and the other is local type. The recent observation shows that Aurelia aurita may belong to the local type since they mainly distribute in Jiaozhou Bay and Qinhuangdao coastal sea, replenish themselves and complete their life history; Nemopilema nomurai and Cyanea nozakii may belong to the foreign type, because their larvae were not found in these places during the past winter and spring. That agrees with the observation and study of Wang et al. and Sun et al.[4-5]. Here, only large jellyfish of foreign type is discussed in the following section.

Scientists analyze the key dynamic mechanism of jellyfish drift and accumulation with numerical models. Barz et al.[6] found the advection process played an important role in the distribution of jellyfish in the central Baltic Sea. Without considering tidal movement in ROMS (Regional Ocean Model System), Moon et al.[7] showed the jellyfish distribution was affected by the coastal current and wind stress between East China Sea and Japan Sea. However, Luo et al.[8] opposed Moon's view with considering the tidal movement in ocean model. Johnson et al.[9] indicated that the seasonal variation of circulation played an important role in the distribution and abundance of jellyfish.

Graham et al.[10] showed the 'Diurnal Vertical Migra- tion' (DVM) phenomena was common in jellyfish of hydromedusae, Scyphozoa and Siphonophorae. However, in 2006 Yellow Sea survey cruises, Zhang et al. [11] found that the number changes of jellyfish in 24 hours is not caused by DVM. During 2006 2007 bottom trawl survey, large jellyfish appeared in many samples of surface or subsurface layers. In the jellyfish survey of Jiaozhou Bay, DVM is not obvious from August to September. In southern Japan Sea, Nemopilema nomurai mainly distributed in the waters shallower than $40 \mathrm{~m}$, and its distributed depth at night is often greater than that at day. According to the observation of 2011 2013 near the coastal waters of Qingdao and Qinhuangdao, jellyfish are found on the surface at day. Therefore, the autonomic movement of different or the same kind of jellyfish under different conditions is not the same, which may be related to light or not. The mechanism of jellyfish autonomic movement is vague. The main distribution depth of jellyfish is not the same. Hence, with reference to the ensemble forecasting concept, an independent ensemble model of large jellyfish would be developed to simulate its trajectory quickly.

\section{Model introduction and configuration, data source}

ROMS is a three-dimensional, free-surface, terrainfollowing numerical model that developed as a community model by Rutgers University and has been widely used by the scientific community for a diverse range of applications. According to the location of large jellyfish and the forecasting demand for emergency response, three sub-region dynamic ROMS models are established by using the multi-nested technique (Fig.1). 
Big, middle and small region models cover northwestern Pacific, Yellow and Bohai Sea, and the coastal waters of Qingdao and Qinhuangdao with increasing resolution, respectively. The surface forcing parameters are supplied by operational Weather Research and Forecasting Model (WRF). The initial and boundary fields of big-region model such as elevation, current, temperature and salinity are obtained from the operational forecasting dataset (HYCOM+NCODA Global $1 / 12^{\circ}$ Analysis). The tidal influence is definitely included in our small-region model by adding the tidal elevation to the sea level height at the open boundaries.

The observation of large jellyfish mainly applies the following methods: fisheries survey, trawl monitoring, visual observation underway period, monitoring by airplane, underwater camera monitoring, sonar monitoring.

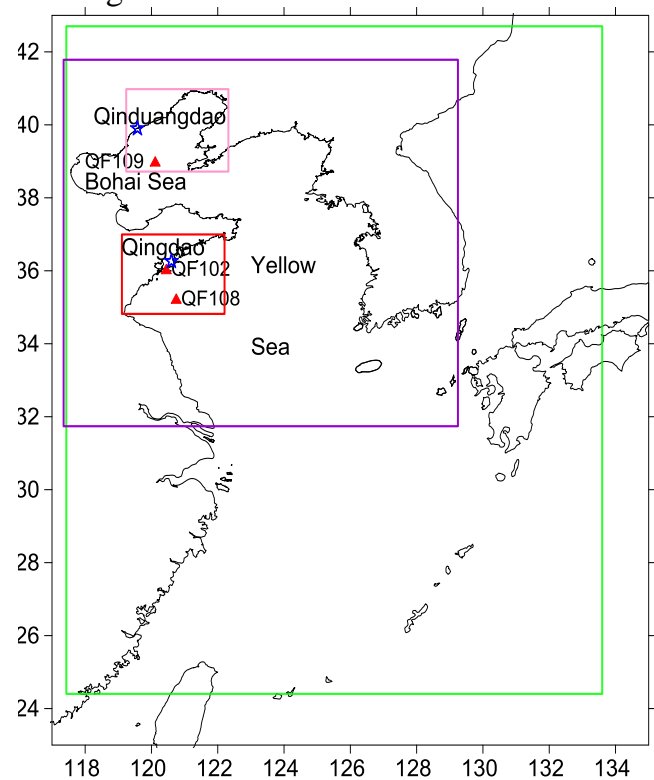

Figure 1. Nested domain of model (purple, red and pink boxes represent the domain of the Yellow and Bohai Sea, the coastal waters of Qingdao and Qinhuangdao, respectively; red triangles represent QF102, QF108 and QF109 buoy).

\section{Ensemble trajectory schemes for large jellyfish}

Because of the characteristics of jellyfish autonomic movement, independent ensemble trajectory model of large jellyfish is developed to simulate its trajectory based on Lagrange particle tracking method and WRF and ROMS results. The WRF and ROMS results is the operational forecast results of marine and atmosphere environment and could be obtained at fixed time every day. Once large jellyfish was found, this model would simulate the ensemble trajectories in short time.

With the vertical movement of large jellyfish taken into account, seven ensemble schemes are designed for Qingdao. Since the water depth near the coastal waters of Qingdao is about $20 \mathrm{~m}$, and the jellyfish maybe distribute between surface and middle layer, the vertical motion amplitude of jellyfish is set to half of the depth and vertical depth from the seabed is not more than $5 \mathrm{~m}$.
1. Without vertical diurnal movement, particles initial released in $0.5 \mathrm{~m}$

2. Without vertical diurnal movement, particles initial released in $1.5 \mathrm{~m}$

3. Particles released in $0.5 \mathrm{~m}$, sinked and located at about half of depth during day (6:00 18:00) and floated and located at surface during night (18:00 6:00)

4. Same as scheme 3 except with particles released in $1.5 \mathrm{~m}$

5. Particles released in $0.5 \mathrm{~m}$, floated and located at about half of the depth during day (6:00 18:00) and sinked and located at surface during night (18:00 6:00)

6. Same as scheme 5 except with particles release in $1.5 \mathrm{~m}$

7. Same as scheme 3 except with located at deeper than half of the depth

Since the water depth there is relatively shallow, only the first five identical schemes are implemented for Qinhuangdao.

\section{Meteorological and marine model validation}

The observation of QF102 in the Yellow and Bohai Sea such as wind at 10 meter and ocean current agrees well with the model results (Fig. 2 3). So does the QF108 and QF109 buoy (figure omitted). For QF102 and QF108 buoy near Qingdao, the forecasting errors of wind speed (direction) of $48 \mathrm{~h}$ is less than $1.77 \mathrm{~m} / \mathrm{s}\left(35.2^{\circ}\right)$, the (relative) forecasting errors of ocean current speed (direction) of $48 \mathrm{~h}$ is less than $19 \%\left(28^{\circ}\right)$ (Tab. omitted). For QF109 buoy near Qinhuangdao, the 48h forecasting errors of wind speed (direction) is less than $1.61 \mathrm{~m} / \mathrm{s}$ $\left(19.2^{\circ}\right)$, the (relative) $48 \mathrm{~h}$ forecasting errors of ocean current speed (direction) is less than 13\% (17\%) (Tab. omitted). The results indicate that the meteorological and marine simulated results are reasonable and reliable in the Yellow and Bohai Sea.
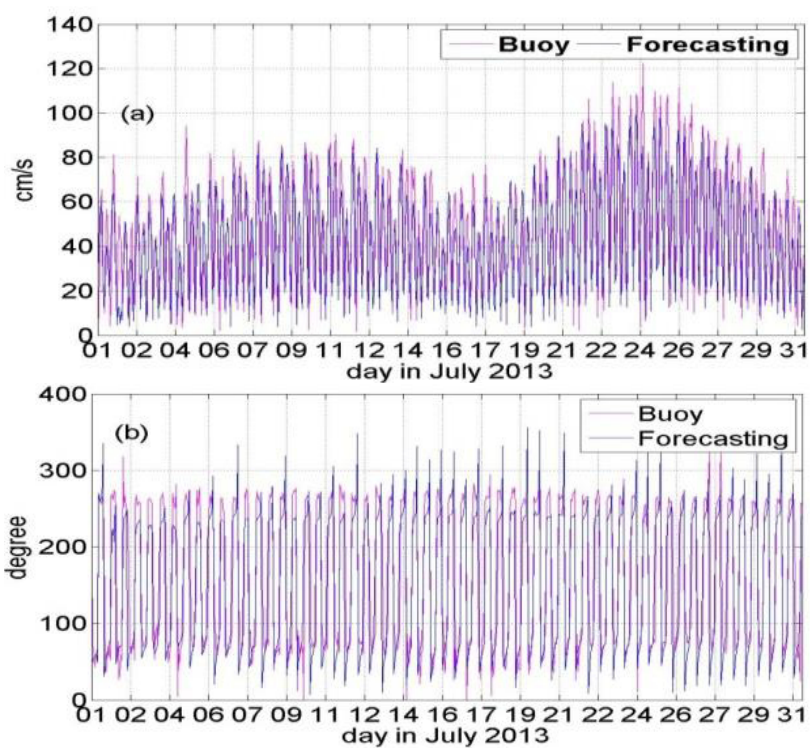

Figure 2. Comparison of surface ocean current speed and direction of QF102 buoy in July 2013. 

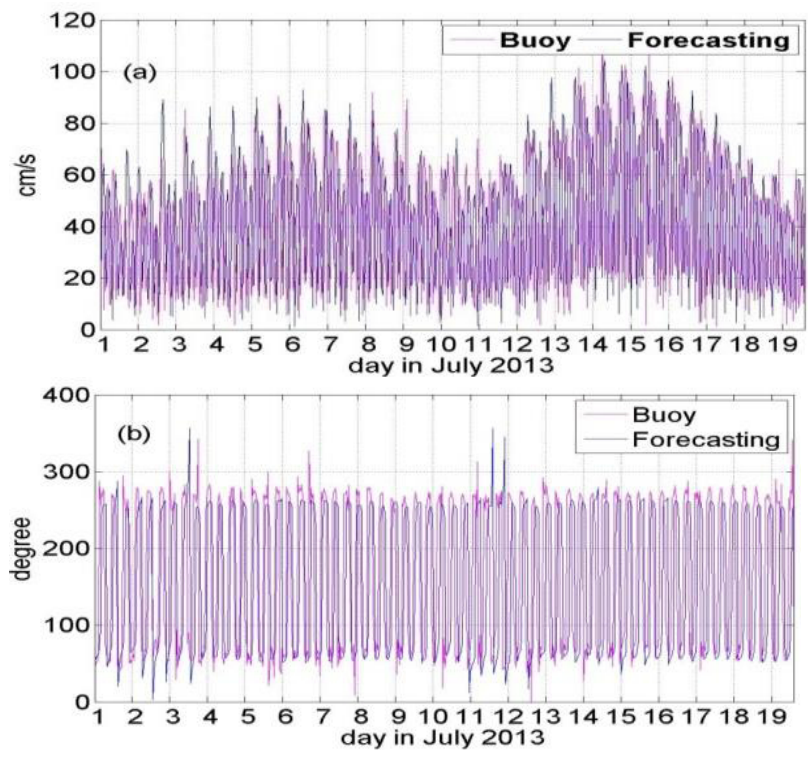

Figure 3. Same as Fig. 2 except with ocean current at $10 \mathrm{~m}$.

\section{Ensemble Trajectory Simulation Results and Validation}

\subsection{Simulation in the Coastal Waters of Qingdao}

With the observation of large jellyfish (Nemopilema nomurai) on July 5th 2012 as the initial state (Fig. 4, green triangle), the ensemble model of jellyfish could simulate the trajectory (Fig.4, blue line), velocity, trend, as well as influenced range (Fig.4, purple line) in about $15 \mathrm{~min}$.

In order to the factors that influence the jellyfish net displacement, 24 hours of low pass filter on the ocean current at surface and middle layers is conducted to filter out the periodical tidal current. As you can see from Fig. 5 , from 5th to 12th July, 2012, the mean wind is southeastward with $2 \sim 3 \mathrm{~m} / \mathrm{s}$ residual ocean current is northward with $0.2 \sim 0.3 \mathrm{~m} / \mathrm{s}$; ocean current speed at midlayer is less than surface layer with counterclockwise deflection. With the influence of strong southeastward wind (figure omitted) and northward surface mean circulation (Fig. 5), Nemopilema nomurai observed in July 5th 2012 moved northward with $5 \mathrm{~km} / \mathrm{d}$ and may affect the waters near Grand Island in future days. Then, the simulated results at 7:50 on July 2 th were compared with observation (Fig. 4, blue triangle), the relative errors of jellyfish drift path ranged from $5.2 \%$ to $24.1 \%$, which indicated that jellyfish observed near Dagong Island might drift from where jellyfish observed on July 5 th.

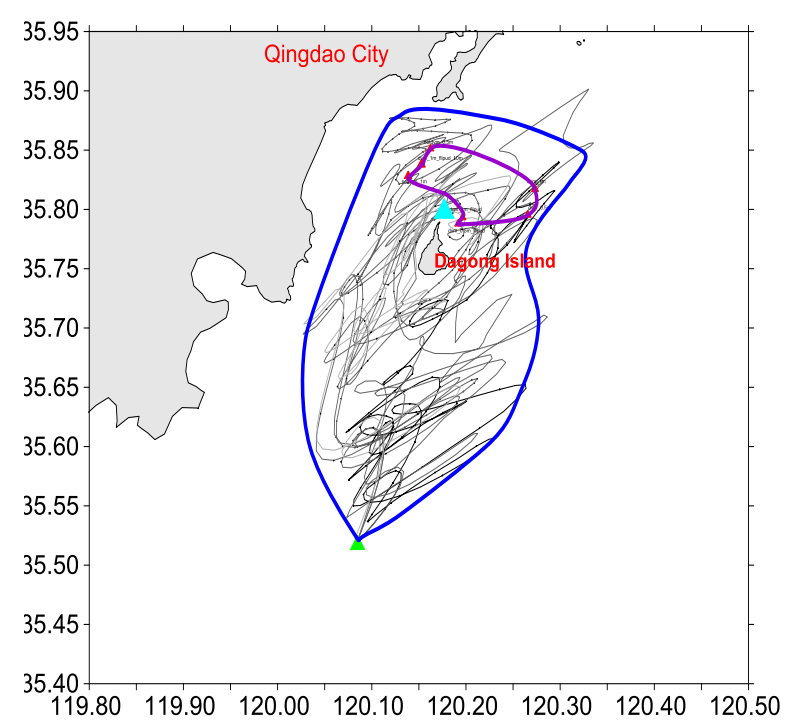

Figure 4. Ensemble trajectories (black lines) from 5th (green triangle) to 12th July 2012 and potential affecting domain (blue line), green(blue) triangle represents nemopilema nomurai monitoring on 11:16 5th (7:50 12th) July, respectively. The region surrounded by purple lines represents the ensemble results on 7:50 July 12 th.

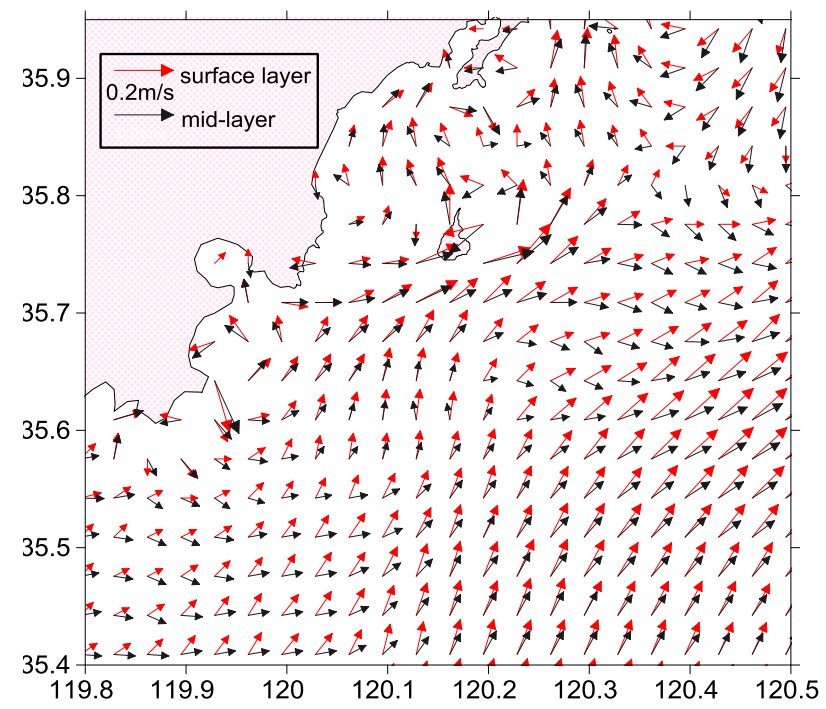

Figure 5. Averaged wind at $10 \mathrm{~m}($ unit $: \mathrm{m} / \mathrm{s})$ and residual ocean current (unit :m/s) at surface and middle layerfrom 5th to 12th July of 2012

On July 18th of 2013, a large number of nemopilema nomurai were observed in the coastal waters from Huangdao to Jiaonan, Qingdao. Based on seven ensemble schemes, the ensemble model was implemented to simulate the drift trajectories. The results showed that due to the strong southerly winds (figure omitted) and northward ocean current (Fig.6), large jellyfish moved northward, influenced the coastal waters of XueJia Island, passed the mouth of Jiaozhou Bay, and then entered the Jiaozhou Bay. Large jellyfish moved with velocity about $8 \mathrm{~km} / \mathrm{d}$. The ensemble trajectories from 18th to $21 \mathrm{st}$ July and influenced range at different times are shown in Fig. 7. 


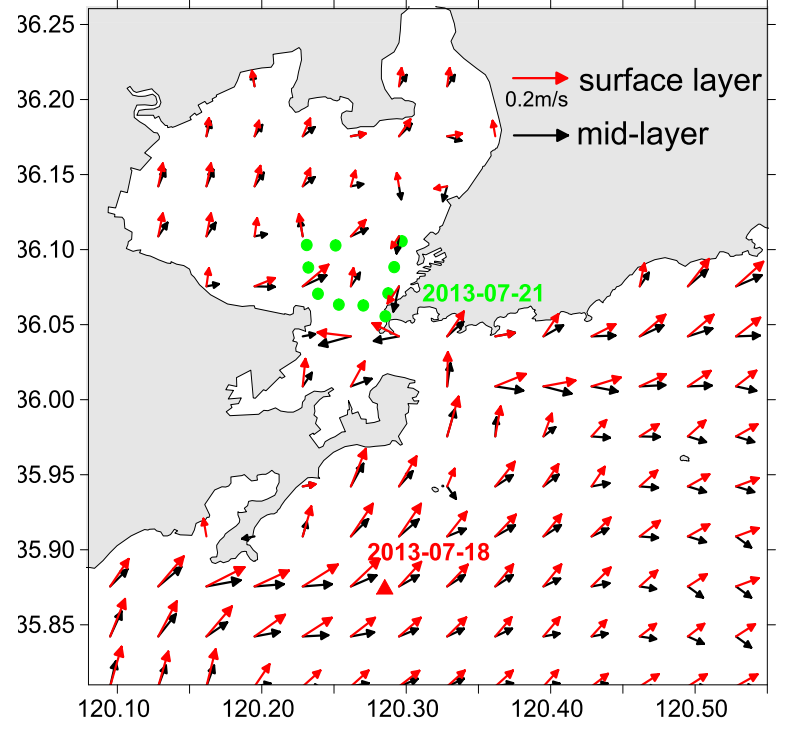

Figure 6. Same as Fig.5 except from 18th to 21st July 2013 (red triangle represents the observation on 18th July while green dots represent the observation at different times of 21st July)

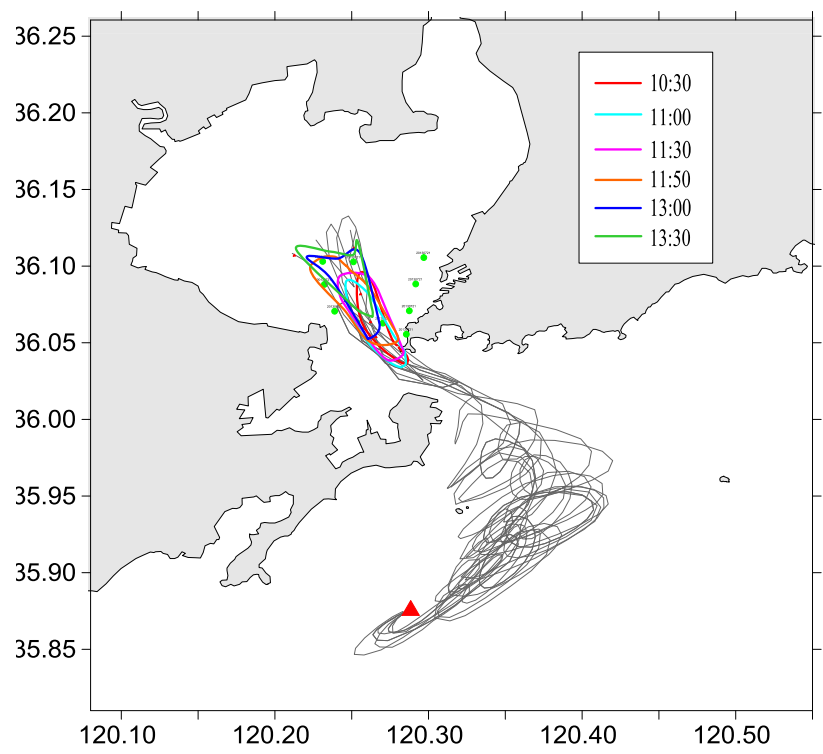

Figure 7. Ensemble trajectories (black lines) from 18th to $21 \mathrm{st}$ July 2013 and influenced ranges at different times

As we can see, influenced ranges characterized by different shapes at different times, while moves northeastward with influence of northward-northeastward current in Jiaozhou bay. The relative errors of drift distance are less than $28 \%$ (Tab. 1).

Table 1. Relative error of drift distance of large jellyfish at different times

\begin{tabular}{c|c|c|c}
\hline Date & Time & $\begin{array}{c}\text { Minimum } \\
\text { relative } \\
\text { error(\%) }\end{array}$ & $\begin{array}{c}\text { Maximum } \\
\text { relative } \\
\text { error(\%) }\end{array}$ \\
\hline \multirow{4}{*}{21 th } & $10: 30-10: 35$ & 13.9 & 27.5 \\
\cline { 2 - 4 } July, & $11: 00-11: 05$ & 10.7 & 27 \\
\cline { 2 - 4 } 2013 & $11: 30-11: 35$ & 5.1 & 16.4 \\
\cline { 2 - 4 } & $11: 50-11: 55$ & 4.1 & 16.9 \\
\cline { 2 - 4 } & $13: 00-13: 05$ & 5 & 19.3 \\
\hline
\end{tabular}

\subsection{Ensemble simulation in the coastal Waters of Qinhuangdao}

The ensemble trajectories from 21 st to 25th July and its potential influenced ranges (Fig. 8b, blue line) are simulated. The results show that with the effect of weak easterly (figure omitted) and ocean current (Fig.8a), jellyfish moved westward, then moved northwestward and consequently influenced Zhongzhi beach near Beidaihe (Fig.8b). The potential influenced range is almost as the same as the observation of 25th July. The relative errors of drift distance range from $13.5 \%$ to $16.7 \%$.
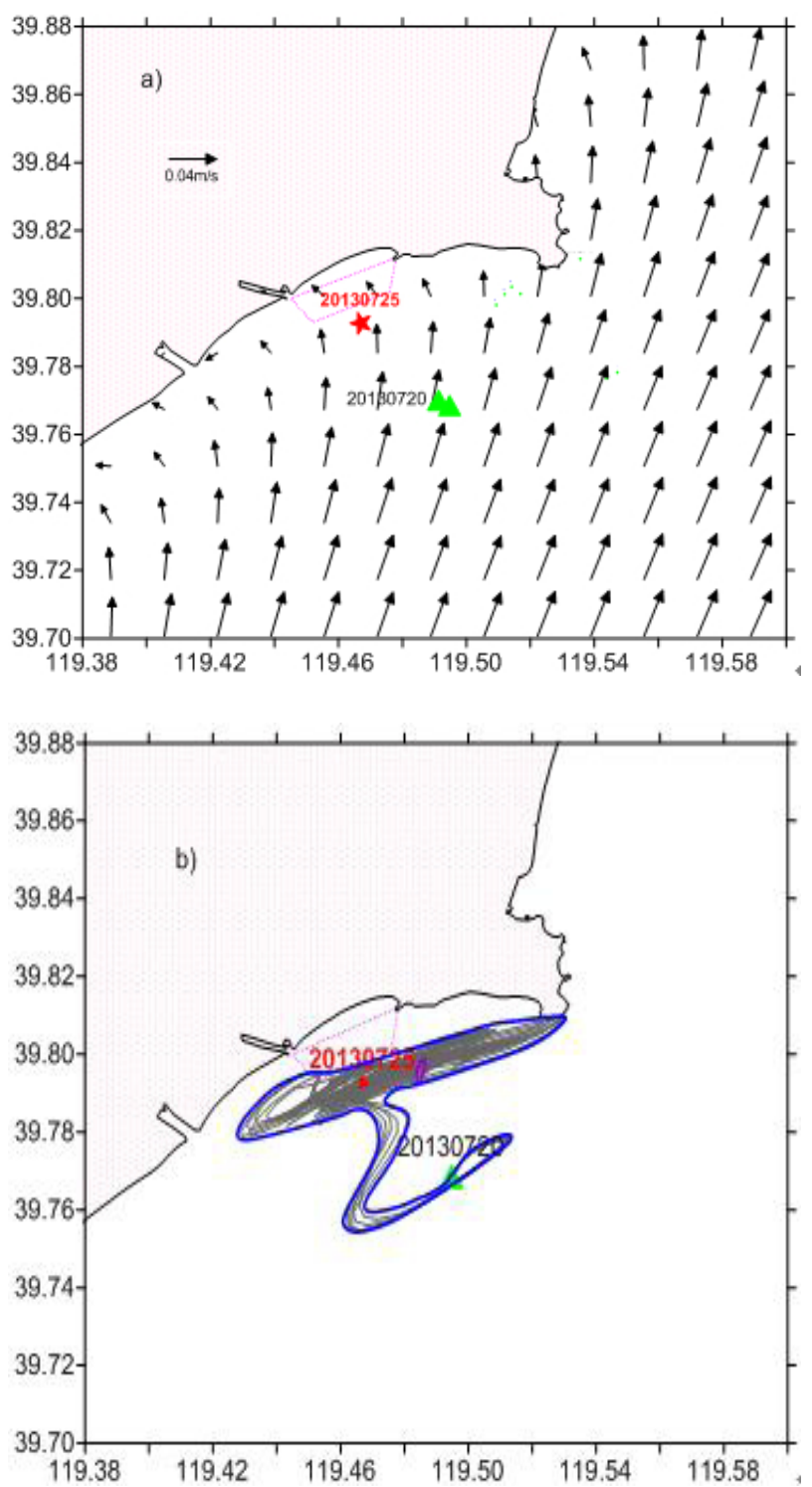

Figure 8. Depth-averaged residual ocean current from 21 st to 25th July 2013(a), ensemble drift trajectory (black line) from 21 st (green triangle) to 25th July 2013 of jellyfish and potential affecting domain (blue line)(b).

\section{Summary and Discussion}

In recent years, in addition to Red Tide and Green Tide, one ecological disaster induced by mass propagation of jellyfish starts to get attention in global. Since the vertical movement vary largely, and its mechanism is vague. 
Hence, we apply the ensemble forecasting concept to simulate the trajectory of jellyfish, with its living habits taken into account. The coastal waters of Qingdao and Qinhuangdao are chosen as the typical jellyfish disaster places of the Bohai and Yellow Sea. Different schemes are designed according to the different environment parameters related to the vertical movement of jellyfish, such as ocean topography or different habits of vertical movements.

Besides, this ensemble model for jellyfish trajectories is independently developed, based on operational forecast results of marine and atmosphere environment, in which seven (five) different ensemble schemes are adopted. And we could simulate the ensemble trajectory in short time because the oceanic dynamical results could be operationally obtained at fixed time every day. Once large jellyfish was found, multi-source observation would be integrated, and this model would simulate the ensemble trajectories in short time.

This model can deliver ensemble simulation of trajectory, direction and potential influenced ranges. The comparisons between monitoring and numerical simulation results in 2012 and 2013 indicate that our ensemble model is suitable for description of jellyfish movements and can behave very well. Compared to single trajectory model, our ensemble model can present more accurate information of potential influenced range, which provides scientific decision support for emergency disposal and management of jellyfish disaster.

\section{Acknowledgements}

This work is supported by the Marine Public Foundation of State Oceanic Administration (No. 201005018) and open found of Key Laboratory of Ocean Circulation, Institute of Oceanology, Chinese Academy of Science (KLOCAW1403). Many thanks for the reviewers' helpful comments.

\section{References}

1. Brodeur R D, Mills C E, Overland J E, et al. Evidence for a substantial increase in gelatinous zooplankton in the Bering Sea, with possible links to climate change[J]. Fish Oceanogr., 1999, 8(4): 296306.

2. Mills C E. Jellyfish blooms: are populations increasing globally in response to changing ocean conditions? [J]. Hydrobiologia, 2001, 451: 55-68.

3. Nagai T. Recovery of fish stocks in the Seto Inland Sea[J]. Mar. Pollut. Bull., 2003, 47: 126-131.

4. Sun, S., 2012. New perception of jellyfish bloom in the east china sea and yellow sea[J], Oceanologia et Limnologia Sinica, 43(3), 406-410.

5. Wang S W, Zhang G T and Sun S. et al., 2012. Population dynamics of three scyphozoan jellyfish species during summer of 2011 in jiaozhou bay[J], Oceanologia et Limnologia Sinica, 43(3), 471-479.

6. Barz K, Hinrichsen H H, and Hirche H J, 2006. Scyphozoa in the Bornholm basin, (central Baltic
Sea)--The role of advection[J]. J. Mar. Syst., 60(1-2), 167-176.

7. Moon, J. H., Pang, I. C., and Yang, J. Y., et al., 2010. Behavior of the giant jellyfish Nemopilema nomurai in the East China Sea and East/Japan Sea during the summer of 2005: A numerical model approach using a particle-tracking experiment [J]. Journal of Marine Systems, 80: 101-114.

8. Luo, X. F., Wei, H., Wang, Y. H., 2012. Processes influencing jellyfish particle tracking in the Yellow Sea and East China Sea[J], Oceanologia et Limnologia Sinica, 43(3): 635-642.

9. Johnson, D. R., Perry, H. M., and Burke, W. D., 2001. Developing jellyfish strategy hypotheses using circulation models[J]. Hydrobiologia, 451: 213-221.

10. Graham, W. M., Pagès, F., and Hamner, W. M., 2001. A physical context for gelatinous zooplankton aggregations: a review[J]. Hydrobiologia, 451: 199212.

11. Zhang F, Zooplanktivorous Gelatinous Taxa: Medusas in the Yellow Sea and East China Sea[D], Qingdao, doctoral dissertation, 2008,1-130. 